\title{
CATETERIZAÇÃO DE SEIO PETROSO INFERIOR NO DIAGNÓSTICO DE SÍNDROME DE CUSHING
}

\section{INFERIOR PETROSAL SINUS SAMPLING IN THE DIAGNOSIS OF CUSHING'S SYNDROME}

Vanessa Cristine Ribeiro Friedrich ${ }^{1}$, Felipe Wolff Schwambach ${ }^{1}$, Karina Zanlorenzi Basso Manosso ${ }^{1}$, Leilane Vensão ${ }^{1}$, Nicole Balster Romanzini ${ }^{1}$, Sthefanie Giovanna Pallone ${ }^{1}$, Thayse Graciella Lozovoy ${ }^{1}$, César Luiz Boguszewski ${ }^{1}$

\section{RESUMO}

Introdução: 15\% dos casos de síndrome de Cushing ACTH-dependente devem-se a tumor ectópico. Técnicas bioquímicas e radiológicas têm sido desenvolvidas para ajudar na distinção entre produção ectópica ou hipofisária de ACTH. Objetivo: Relato de síndrome de Cushing ACTH-dependente, cuja definição diagnóstica foi realizada por cateterização bilateral de seio petroso inferior. Relato de caso: Mulher, 47 anos, com amenorreia, ganho de peso, fraqueza, hipertensão arterial, osteoporose e transtorno de humor. Ao exame, fácies em lua cheia e giba dorsal, estrias violáceas abdominais de 1,5 cm, membros inferiores com edema. A investigação laboratorial detectou hipercortisolismo ACTH-dependente. Teste de supressão com dexametasona $(16 \mathrm{mg}$ ) sugeriu produção ectópica de ACTH. Entretanto, exames de imagem não detectaram lesões no sistema nervoso central ou fora dele responsáveis pelo excesso hormonal. A paciente foi encaminhada a outro serviço para realização de cateterização de seio petroso inferior, que não evidenciou gradiente entre os níveis de ACTH dosados próximo à glândula comparados à periferia, confirmando a origem ectópica do ACTH. Novos exames de imagem evidenciaram tumor em timo. Paciente foi submetida à videotoracoscopia com exérese da lesão, recebendo alta no $10^{\circ}$ pós operatório com normalização dos níveis de cortisol e ACTH. Conclusão: A cateterização bilateral de seio petroso inferior é o teste que oferece maior sensibilidade e especificidade na diferenciação entre produção hipofisária e ectópica de ACTH. Uma vez que os exames de imagem iniciais não evidenciaram tumor, esse teste foi essencial na confirmação da etiologia ectópica do ACTH, o que motivou nova investigação e, consequente, diagnóstico e tratamento.

Palavras-Chaves: Síndrome de Cushing. Síndrome de ACTH ectópico. Amostragem do seio petroso.

\section{ABSTRACT}

Introduction: 15\% of ACTH-dependent Cushing's syndrome is due to ectopic ACTH-secreting tumor. Radiological and biochemical techniques have been developed to assist distinguish the source of the hormone: ectopic or pituitary gland. Objective: Case report of an ACTH-dependent Cushing's syndrome whose diagnostic definition was conducted by bilateral inferior petrosal sinus sampling. Case Report: A 47 years old female, presenting with amenorrhea, weight gain, weakness, hypertension, osteoporosis, mood disorder. On examination, moon facies, dorsal hump, abdominal purple striae $1.5 \mathrm{~cm}$ of width, lower limb edema. Laboratory findings demonstrated ACTH-dependent hypercortisolism. Suppression test with dexamethasone $16 \mathrm{mg}$ suggested ectopic ACTH production. However, imaging did not detect lesions in the CNS or elsewhere responsible for hormone excess. The patient was referred to another service to perform inferior petrosal sinus sampling, which showed no gradient between the ACTH levels measured close to the gland compared to the periphery, confirming the ectopic source of ACTH. New imaging tests showed tumor in the thymus. Patient underwent thoracoscopy with excision of the lesion and was discharged on the 10th postoperative with normalization of cortisol and ACTH. Conclusion: The bilateral inferior petrosal sinus sampling is the test that provides greater sensitivity and specificity in differentiating pituitary and ectopic ACTH production. Once the initial imaging tests showed no tumor, this test was instrumental in confirming the etiology of ectopic ACTH, which led to new research and, consequently, diagnosis and treatment.

Keywords: Cushing syndrome. ACTH syndrome, ectopic. Petrosal sinus sampling.

1-Universidade Federal do Paraná

Contato do Autor / Mail to:

Vanessa Cristine Ribeiro Friedrich - vaneribeiro@gmail.com 


\section{INTRODUÇÃO}

A suspeita clínica de síndrome de Cushing requer a confirmação laboratorial do hipercortisolismo e investigação de sua origem. Entretanto, dado a taxa de falsos positivos e negativos dos diversos testes, muitas vezes o diagnóstico só é realizado com repetição e associação de exames. Estes, por sua vez, se baseiam no estímulo ou inibição do eixo hipotálamo-hipófise-adrenal. Aproximadamente 15\% dos casos de síndrome de Cushing ACTH-dependente devem-se a tumor ectópico. Técnicas bioquímicas e radiológicas têm sido desenvolvidas para ajudar na distinção entre produção ectópica ou hipofisária de ACTH. $^{1}$ Exames de imagem e técnicas utilizando isótopos têm papel importante na localização da fonte de ACTH ectópico, mas nem sempre são eficazes. ${ }^{2}$

\section{OBJETIVO}

Relato de caso de síndrome de Cushing ACTHdependente cuja definição diagnóstica foi realizada com ajuda de cateterização de seio petroso inferior.

\section{RELATO DE CASO}

Paciente feminina, 47 anos, apresentando queixa de amenorreia há 3 anos, ganho de peso de cerca de 15 kg, fraqueza generalizada, hipertensão arterial, osteoporose e transtorno de humor. Negava uso de corticosteroides sistêmicos ou tópicos. Ao exame, hipertensa, fácies em lua cheia e giba dorsal, estrias violáceas abdominais de $1,5 \mathrm{~cm}$, membros inferiores com edema $+/ 4$. Os exames laboratoriais revelaram: $\mathrm{Hb} / \mathrm{VG}$ 13,2/40,9; leucócitos 11000; HbA1C 5,7\%; glicemia 84; TSH 1,07; creatinina 0,7 ; ureia $15 \mathrm{mg} / \mathrm{dl}$; sódio $141 \mathrm{e}$ potássio 3,7 meq/l. O cortisol livre urinário foi de 1050 $\mu \mathrm{g} / 24 \mathrm{~h}$ (valor de referência 55 - 286), cortisol plasmático pós dexametasona $1 \mathrm{mg}$ de 29,7 $\mathrm{\mu g} / \mathrm{dl}$ (valor esperado $<2$ ). A dosagem de ACTH foi de $43,3 \mathrm{pg} / \mathrm{ml}$ (valor de referência 10-50). Uma vez estabelecido hipercortisolismo ACTH-dependente, foram realizados os seguintes exames radiológicos: RNM hipófise: normal; TC tórax: fratura de arcos costais à esquerda; TC abdome e pelve com hiperplasia adrenal bilateral. Foi realizado teste com dexametasona $16 \mathrm{mg}$ em $48 \mathrm{~h}$ havendo supressão de apenas 30\% em relação ao basal, sugerindo produção ectópica de ACTH. Paciente tratada com cetoconazol 200 mg 8/8h e ácido zolendrônico. Foi encaminhada a outro serviço para realização de cateterização de seio petroso inferior, 0 qual evidenciou gradiente ACTH central/periférico basal $<2$ e gradiente ACTH central/periférico após DDAVP $<3$, confirmando a origem ectópica do ACTH. Ao repetir exames de imagem para localização de tumor, TC tórax evidenciou lesão em timo medindo $18 \times 34 \mathrm{~mm}$. Submetida a videotoracoscopia com exérese de lesão, recebeu hidrocortisona $100 \mathrm{mg}$ 8/8h no pós operatório, com progressivo desmame de corticoide e anti-hipertensivos. Paciente recebeu alta no $10^{\circ}$ dia PO com normalização de cortisol e ACTH.

\section{DISCUSSÃO}

A primeira etapa no diagnóstico laboratorial da síndrome de Cushing é a documentação do hipercortisolismo endógeno. Para tal, realiza-se dosagem de cortisol livre urinário, salivar ou sérico, também sendo possível a avaliação de supressão após dexametasona em baixa dose $(1 \mathrm{mg})^{3}$. A causa mais comum de hipercortisolismo é exógena, situação na qual cortisol e ACTH encontram-se suprimidos. No caso relatado, a confirmação do hipercortisolismo endógeno se deu por dosagens de cortisol livre urinário e a ausência de supressão com dexametasona em baixa dose. Para estabelecer o diagnóstico etiológico da síndrome de Cushing, realiza-se dosagem do ACTH e testes de supressão do cortisol com altas doses de dexametasona. Níveis indetectáveis de ACTH plasmático $(<10 \mathrm{pg} / \mathrm{ml})$ confirmam a síndrome de Cushing decorrente de um tumor adrenal produtor de cortisol. ${ }^{3}$ Níveis de ACTH situados na faixa inferior da normalidade (entre 10 e 20 pg/ml) devem ser repetidos. Por outro lado, um ACTH inapropriadamente elevado para os níveis de cortisol, como no caso clínico em questão, apontam para síndrome de Cushing ACTHdependente: doença de Cushing (tumor hipofisário) ou síndrome do ACTH ectópico. Para a elucidação da fonte do ACTH, inicialmente o teste de supressão com altas doses de dexametasona (16 mg em 48h) apontou para produção ectópica (supressão $<50 \%$ basal). Este teste baseia-se na persistência de feedback negativo pelos adenomas hipofisários produtores de ACTH em resposta aos glicocorticóides, porém com a curva dose-resposta deslocada para direita, ou seja, a supressão só ocorre após altas doses de dexametasona 4 . Uma vez que exames de imagem não demonstraram uma fonte possível de ACTH ectópico, tampouco lesão em SNC confirmando tratar-se de doença de Cushing, optou-se pela realização de cateterização bilateral do seio petroso inferior com dosagem de cortisol e ACTH após administração de DDAVP. Esse é um procedimento de intervenção radiológica no qual os niveis de ACTH obtidos da drenagem venosa bem próxima da hipófise são comparados com os níveis sanguíneos periféricos antes e após estímulo com $\mathrm{CRH}$ (ou DDAVP). Um gradiente entre essas duas localizações indica Cushing hipofisário, enquanto a ausência de gradiente, como no caso relatado, sugere origem ectópica. ${ }^{1}$ É o teste que oferece a mais alta sensibilidade (96-100\%) e especificidade (100\%) para tal. Sua sensibilidade é aumentada quando associado ao estímulo com $\mathrm{CRH}$, uma 
vez que no adenoma de hipófise os corticotrofos respondem com um incremento na liberação do ACTH em $35 \%$ e do cortisol em $20 \%{ }^{5}$. Já os tumores ectópicos e os tumores adrenais não respondem ao $\mathrm{CRH}$, pois apresentam supressão dos corticotrofos.

\section{CONCLUSÃO}

A cateterização bilateral de seio petroso inferior é o teste que oferece maior sensibilidade e especificidade na diferenciação entre produção hipofisária e ectópica de ACTH. A ausência de gradiente entre os níveis de ACTH dosados próximo à glândula comparados à periferia confirma Cushing ectópico. Uma vez que os exames de imagem iniciais não evidenciaram tumor, esse teste foi essencial na confirmação da etiologia ectópica do ACTH, o que motivou nova investigação e, consequente, diagnóstico e tratamento. 0 cateterismo bilateral do seio petroso inferior é aceito como etapa importante no protocolo de investigação das causas de síndrome de Cushing ACTH-dependentes, mas seu uso ainda é restrito a um número limitado de serviços no Brasil.

\section{REFERÊNCIAS}

1. Utz A, Biller MKB. The role of bilateral inferior petrosal sinus sampling in the diagnosis of Cushing's syndrome. Arq Bras Endocrinol Metab 2007;51/8

2. Santos S, Santos E, Gaztambide S, Salvador J. Diagnosis and differential diagnosis of cushing's syndrome. Endocrinol Nutr. 2009;56(2):71-84

3. Castro M, Moreira AC. Diagnóstico Laboratorial da Síndrome de Cushing. Arq Bras Endocrinol Metab 2002;46/1; 97-105

4. Liddle GW. Tests of pituitary-adrenal suppressibility in the diagnosis of Cushing's syndrome. J Clin Endocrinol Metab 1960;20:1539-60.

5. Nieman LK, Oldfield EH, Wesley R, Chrousos GP, Loriaux DL, Cutler GB Jr. A simplified morning ovine corticotropin-releasing hormone stimulation test for the differential diagnosis of adrenocorticotropindependent Cushing's syndrome.J Clin Endocrinol Metab 1993;77:1308-12. 